\title{
CONTROL DE LA DINÁMICA FOLICULAR OVÁRICA EN LLAMAS (LAMA GLAMA) EN POSPARTO TEMPRANO
}

\author{
CONTROL OF OVARIAN FOLLICULAR DYNAMICS IN EARLY POSTPARTUM LLAMA \\ (LAMA GLAMA)
}

\begin{abstract}
Cancino, A.K. ${ }^{1}$, Aller, J.F. ${ }^{2 *}$, Rebuffi, G. ${ }^{3}$ y Alberio, R.H. ${ }^{2}$
${ }^{1}$ Instituto Nacional de Tecnología Agropecuaria. EEA Bariloche. Río Negro. Argentina. akcancino@bariloche.inta.gov.ar

${ }^{2}$ Instituto Nacional de Tecnología Agropecuaria. EEA Balcarce. Balcarce. Argentina. *jaller@balcarce.inta.gov.ar

${ }^{3}$ Facultad de Veterinaria. Universidad Católica. Salta. Argentina. grebuffi@yahoo.com.ar
\end{abstract}

\section{Palabras CLAVE ADICIONALES}

Camélidos sudamericanos. Folículo dominante. Ovulación.

\section{RESUMEN}

Se evaluó el efecto del acetato de medroxiprogesterona (AMP, esponja intravaginal) con o sin inyección de benzoato de estradiol (BE) sobre el patrón de regresión folicular, la emergencia de la onda folicular y la tasa ovulatoria en llamas en posparto temprano y sin cría. El trabajo se realizó en la Estación Experimental de Altura del INTA de Abra Pampa, ubicada a $3484 \mathrm{msnm}$. Un total de 48 Ilamas adultas fueron categorizadas en lactancia temprana $(n=24,30 \pm 4$ días posparto, media $\pm D E)$ y sin cría $(n=24)$. Cada hembra fue distribuida de acuerdo a la fase folicular ( $F F=$ crecimiento, estática o regresión) del folículo dominante (FD1) determinada por ultrasonografía ovárica desde 3 días previos al tratamiento. El día 0 , todas las hembras recibieron una esponja intravaginal (150 mg de AMP) durante 8 días más 5 mg AMP (im) y la mitad de las hembras recibió aleatoriamente 2 mg de BE (im). Desde el día 0 hasta el 14, la respuesta ovárica fue monitoreada diariamente por ecografía. El día 12, las hembras que presentaron un folículo dominante $\geq 6 \mathrm{~mm}$ (FD2) de una nueva onda folicular fueron tratadas con $\mathrm{GnRH}$ (8 $\mu \mathrm{g}, \mathrm{im})$ para inducir la ovulación. Se midió la concentración plasmática de $17 \beta$-estradiol (desde el día 0 al 8) y progesterona (día 14) para confirmar la ovulación. Se utilizó un diseño factorial $2 \times 2 \times 3$ (estado lactacional, tratamiento con o sin BE y fases foliculares). Se observó interac-

\section{AdDitional KEYWORDS}

South American camelids. Dominant follicle. Ovulation.

ción entre el estado lactacional y la fase folicular sobre el patrón de regresión del FD1, atribuida a una mayor duración en hembras sin cría ( 6,0 días) que en las hembras con cría (3,5 días) durante la fase estática. El día de emergencia del FD2 fue diferente $(p<0,05)$ entre tratamientos (con $B E=$ día $6,5 \pm 0,6$ y $\sin B E=$ día $4,2 \pm 0,5$ ). El diámetro del FD2 al día 12 fue afectado $(p<0,05)$ por el tratamiento (con $B E=6,4 \pm 0,3 \mathrm{~mm}$ vs. $\sin \mathrm{BE}=8,2 \pm 0,3 \mathrm{~mm}$ ) y el estado lactacional (sin cría $=8,0 \pm 0,4 \mathrm{~mm}$ vs. lactancia temprana $=6,6 \pm 0,3 \mathrm{~mm}$ ). La tasa ovulatoria total (hembras con ovulación/hembras tratadas) obtenida en el tratamiento con BE $(45,8 \%)$ fue menor $(p<0,05)$ que en el tratamiento sin $B E$ $(82,6 \%)$. En conclusión, el tratamiento con AMP (intravaginal e inyectable) combinado con una inyección de $\mathrm{BE}$, demoró la emergencia de una nueva onda folicular en aproximadamente dos días y el diámetro del FD2 al día 12 y la tasa ovulatoria fueron menores que en hembras $\sin \mathrm{BE}$. La lactancia estuvo asociada con desarrollo folicular deprimido, sin afectar al porcentaje de hembras que ovuló posterior al tratamiento con AMP.

\section{SUMMARY}

The effect of treatment with medroxiprogesterone acetate (MPA, intravaginal sponge) combined with or without injection of estradiol 
benzoate (EB) on the patterns of follicular regression, follicular wave emergence and ovulation rate in early postpartum and non-lactating llamas was evaluated. This work was carried out at the Experimental Station of Altitude INTA Abra Pampa (3484 m above sea level). A total of 48 adult females were categorized as lactating $(n=24$; $30 \pm 4$ d postpartum, mean $\pm S D$ ) or non-lactating $(n=$ 24). Each one was distributed according to the follicular phase ( $\mathrm{FP}=$ growing, static or regressing) of dominant follicle (DF1) determined by daily ovarian ultrasonography from 3 days before treatment. At day 0 , intravaginal sponge $(150 \mathrm{mg}$ MPA) was inserted to all llamas for 8 days plus 5 mg MPA (im). Half of the females randomly received $2 \mathrm{mg}$ of EB (im). The ovarian response was monitored daily by ultrasonography from day 0 to day 14 . At day 12 , females having a new dominant follicle $\geq 6 \mathrm{~mm}$ (DF2) were treated with $\mathrm{GnRH}(8 \mu \mathrm{g}$, $\mathrm{im})$ to induce ovulation. Blood samples were collected to determine plasma estradiol-17 $\beta$ (from day 0 to day 8 ) and progesterone (day 14) concentrations to confirm ovulation. Factorial design $2 \times 2 \times 3$ (lactational status, treatment with or without $E B$ and follicular phases) was used. There was lactational status by FP interaction on the regression patterns of DF1 attributed to greater duration in non-lactating females (6.0 days) than for lactating females (3.5 days) in the follicular static phase. The day (mean \pm SEM) of follicular wave emergence was different $(p<0.05)$ between treatments (with $\mathrm{EB}=$ day $6.5 \pm 0.6$ vs. without $\mathrm{EB}=$ day $4.5 \pm 0.5)$. At day 12 , the DF2 diameter $($ mean \pm SEM) was affected $(p<0.05)$ by the treatment (with $\mathrm{EB}=6.4 \pm 0.3 \mathrm{~mm}$ vs. without $\mathrm{EB}=$ $8.2 \pm 0.3 \mathrm{~mm}$ ) and the lactational status (nonlactating $=8.0 \pm 0.4 \mathrm{~mm}$ vs. lactating $=6.6 \pm 0.3 \mathrm{~mm}$ ). The total ovulation rate (females with ovulation/ treated females) in EB-treated females (45.8\%) was lower $(p<0.05)$ than non-treated females $(82.6 \%)$. In conclusion, the MPA treatment (intravaginal plus intramuscular) combined with injection of EB approximately delayed in two days the emergence of a new follicular wave. Therefore, follicular diameter at day 12 and the ovulation rate were lower than no EB treatment. Lactation was associated with depressed follicular development, however, it did not affect the ovulation rate.

\section{INTRODUCCIÓN}

Desde el punto de vista productivo, en un hato de llamas es indispensable lograr una gestación lo antes posible después del parto, para obtener una cría por año de cada hembra en servicio. Por tanto, el uso de las biotécnicas reproductivas en las hembras en lactancia temprana será cada vez más frecuente para aumentar los ingresos de los criadores en el altiplano argentino.

En llamas (Adams et al., 1990), alpacas (Vaughan et al., 2004) y vicuñas (Miragaya et al., 2004), en ausencia de un estímulo ovulatorio, la actividad ovárica ocurre en ondas de crecimiento y regresión folicular. Durante cada onda, un folículo se transforma en dominante (FD), crece hasta su completa madurez y finalmente regresa, o sea, cada onda folicular presenta fases de emergencia, selección, crecimiento, estática y regresión (Adams et al., 1991). El período requerido para completar una onda folicular es aún discutido. Bravo et al. (1990) observaron que dicho intervalo fue de 12 días; en cambio, otros autores, observaron un período de 22-24 días (Adams et al., 1990), 18 días (Aller y Alberio, 1996) y 22,6 días (Chaves et al., 2002).

Varios autores han demostrado el control exógeno del desarrollo folicular mediante la aplicación de progesterona $\left(\mathrm{P}_{4}\right)$ inyectable (Alberio y Aller, 1996) o intravaginal (Chaves et al., 2002). Ambos estudios coincidieron en que el menor diámetro del FD fue observado entre 7 a 8 días después del comienzo del tratamiento, pero sin hacer referencia a la emergencia de una nueva onda folicular. Cavilla et al. (2006) determinaron los perfiles plasmáticos de $\mathrm{P}_{4}$ en un tratamiento con dispositivo intravaginal con $0,78 \mathrm{~g}$ de $\mathrm{P}_{4}$ observando influencia negativa sobre la actividad folicular.

En bovinos, la aplicación de $\mathrm{P}_{4}$ en combinación con estrógenos $\left(\mathrm{E}_{2}\right)$ tuvo un efecto supresivo sobre el desarrollo del FD en fase de crecimiento e indujo la emergencia y desarrollo de un nuevo FD con un ovocito competente (Burke et al., 1998). En llamas, la $\mathrm{P}_{4}$ en combinación con $\mathrm{E}_{2}$ inyectables durante 5 días fue utilizada con éxito para la 
regresión del folículo dominante pero no se observó la emergencia de la nueva onda folicular (Carretero et al., 2006). El uso de progestágenos en esta especie es muy limitado. Aba et al. (1999) determinaron las concentraciones plasmáticas de $\mathrm{P}_{4}$ y $\mathrm{E}_{2}$ naturales en un tratamiento intravaginal con acetato de medroxiprogeterona (AMP), pero no utilizaron la ultrasonografía para observar la dinámica folicular ovárica. Sin embargo, Ferrer et al. (1999) observaron que, esponjas intravaginales impregnadas con AMP desde 120 hasta 480 mg no lograron inhibir ni sincronizar el desarrollo folicular.

Por otro lado, la lactancia está asociada a un menor diámetro máximo del FD (Ratto et al., 2003) y a un menor intervalo entre ondas foliculares sucesivas, sin afectar la tasa de crecimiento del folículo preovulatorio ni el intervalo apareamiento-ovulación (Adams et al., 1990).

No se encontró información sobre el control y sincronización de la onda folicular en llamas en posparto temprano, por lo tanto, es importante conocer la eficiencia de estos tratamientos aplicados en hembras en ese estado reproductivo con el fin de acortar el intervalo parto-gestación.

El objetivo del presente trabajo fue evaluar el efecto del tratamiento con AMP (esponja intravaginal e intramuscular) con o sin inyección de benzoato de estradiol (BE) sobre el patrón de regresión folicular, la emergencia de una nueva onda folicular y la tasa ovulatoria en llamas en posparto temprano.

\section{MATERIAL Y MÉTODOS}

El experimento se llevó a cabo en el rebaño de llamas de la Estación Experimental de Altura del INTA en Abra Pampa (Jujuy, Argentina), ubicada en el Departamento de Cochinoca a una altitud de $3484 \mathrm{msnm}$. (22 $49^{\prime}$ S, $65^{\circ} 47^{\prime} \mathrm{O}$ ) durante la estación de lluvia (marzo-mayo). Las llamas utilizadas fueron seleccionadas teniendo en cuenta los siguientes criterios: mayores de tres años, adecuado tamaño y apariencias no patológicas del tracto reproductivo al examen rectal. Los animales se encontraban en pastoreo sobre pastura natural compuesta por chillagua (Festuca scirpifolia), totorilla (Juncus baltiens) y pasto vicuña (Deyeuxia nardifolia). En el momento de iniciar el tratamiento (día 0), los animales con cría (grupo A, n= 24; 103,5 \pm 11 kg; $30 \pm 4$ días posparto; media $\pm \mathrm{DE}$ ) y sin cría (grupo Bcontrol, $n=24 ; 113 \pm 12 \mathrm{~kg}$; media $\pm \mathrm{DE}$ ) fueron distribuidos en tres subgrupos cada uno, de acuerdo a la fase folicular inicial (crecimiento, estática o regresión) del folículo dominante (FD1), determinado por medio de ecografía diaria durante los tres días previos al comienzo del tratamiento. Al día 0 , todas las hembras fueron tratadas con esponjas intravaginales de poliuretano impregnadas con 150 mg de AMP (Farmabase, Italia), que permanecieron durante 8 días, así como con 5 mg de AMP (im). El mismo día, en forma aleatoria, la mitad de las hembras de cada subgrupo recibió 2 mg de BE (im) (Andenex-Chemie, Alemania) disueltos en $2 \mathrm{ml}$ aceite de girasol. Por lo tanto, quedaron conformados los siguientes subgrupos (1 a 6).

Grupo A = hembras con cría al pie $(n=24)$

Subgrupo 1: folículo en fase de crecimiento, con BE $(n=4)$ o sin BE $(n=4)$.

Subgrupo 2: folículo en fase estática, con BE $(n=4)$ o sin BE $(n=4)$.

Subgrupo 3: folículo en fase de regresión, con BE $(n=4)$ o sin BE $(n=4)$.

Grupo B = hembras sin cría al pie $(n=24)$

Subgrupo 4: folículo en fase de crecimiento, con BE $(n=4)$ o sin BE $(n=4)$.

Subgrupo 5: folículo en fase estática, con BE $(n=4)$ o sin BE $(n=4)$.

Subgrupo 6: folículo en fase de regresión, con $B E(n=4)$ o sin $B E(n=4)$.

Las hembras que al día 12 presentaron un folículo dominante de la nueva onda folicular inducida (FD2) con un diámetro $\geq 6$ mm fueron tratadas con $8 \mu$ g de un análogo sintético (Buserelina; Receptal-Hoechst) de la hormona liberadora de gonadotrofinas 
(GnRH), como inductora de la ovulación. Los ovarios de cada hembra fueron monitoreados mediante ultrasonografía (SonoAce 1500, Medison Co, Korea) utilizando un transductor transvaginal de $6,5 \mathrm{MHz}$, desde 3 días previos al comienzo del tratamiento (día -3) hasta 6 días después del retiro del tratamiento (día 14) para confirmar la ovulación. Con las observaciones ecográficas realizadas los días $-3,-2,-1$ y 0 , las hembras fueron distribuidas a los diferentes subgrupos de acuerdo al estadio de desarrollo del FD1 (crecimiento, estática ó regresión). En la mitad de las hembras tratadas con o sin BE se obtuvieron muestras de sangre diariamente desde el día 0 hasta el día 8 para determinar la concentración plasmática de $17 \beta$-estradiol por medio de RIA (Diagnostic Products Corporation, Los Angeles, USA) para uso en bovinos y validado en plasma de llama (Aba et al., 1995). El coeficiente de variación intraensayo fue $<13 \%$ para concentraciones comprendidas entre 5 y $180 \mathrm{pmol} / \mathrm{l}$. La menor cantidad de $\mathrm{E}_{2}$ detectable fue de $6 \mathrm{pmol} / \mathrm{l}$. Al día 20 se tomaron muestras de sangre para determinar la concentración plasmática de $\mathrm{P}_{4}$ mediante la prueba ELISA y confirmar la ovulación observada el día 14 por medio de ecografía. El coeficiente de variación intraensayo fue $<9 \%$ para concentraciones comprendidas entre 0,65 y $0,75 \mathrm{nmol} / \mathrm{l}(0,20$ y $0,23 \mathrm{ng} / \mathrm{ml})$. La sensibilidad de la técnica fue de $0,3 \mathrm{nmol} / \mathrm{l}(0,09 \mathrm{ng} / \mathrm{ml})$.

Se utilizó un diseño completamente aleatorizado con un diseño factorial de $2 \times 2 \times 3$, cuyos factores principales fueron: estado lactacional (EL; con o sin cría), uso de $\mathrm{BE}$ (BE; con o sin $\mathrm{BE}$ ) y fase folicular inicial (FFI; crecimiento, estática o regresión) con 4 unidades experimentales (repeticiones) por tratamiento. Las variables continuas fueron analizadas mediante ANOVA utilizando el procedimiento GLM del paquete estadístico SAS (SAS, 1989). Cuando las variables no cumplieron con alguno de los supuestos necesarios para realizar el ANOVA, sus valores fueron previamente transformados mediante la $\sqrt{X}(\mathrm{X}=$ valor de la variable). Las concentraciones de $\mathrm{E}_{2}$ fueron analizadas como medidas repetidas utilizando el PROC MIXED. Como variable repetida se utilizó el día de muestreo. Las variables categóricas fueron analizadas por medio del PROC CATMOD. El nivel de significación utilizado fue del 5\%.

\section{RESULTADOS}

Del total de llamas tratadas ( $n=48)$, una hembra sin cría al pie correspondiente al subgrupo 6 ( $\sin \mathrm{BE}$ ), fue descartada por muerte durante el ensayo experimental (día 5).

Estadio folicular previo al tratamiento. El diámetro del FD1 al inicio del tratamiento fue 4,2 y 3,4 mm mayor en las hembras sin cría en fase estática y regresión $(p<0,05)$ respectivamente, que en las hembras con cría (tabla I).

Regresión inducida del FD1. Para analizar los factores que afectaron la regresión inducida del FD1, las llamas con y sin cría al pie en fase de regresión natural al inicio del tratamiento (subgrupo 3 y 6 ) y las que presentaron folículo persistente $(n=5)$, fueron excluidas del análisis estadístico. El análisis factorial de los datos transformados, mos-

Tabla I. Diámetro (mm; media $\pm E E M)$ del folículo dominante en diferentes fases foliculares al inicio del tratamiento intravaginal con AMP en llamas con cría (CC) y sin cría (SC). (Dominant follicle diameter ( $\mathrm{mm}$; mean \pm SEM) in different follicular phases at the start of the MPA intravaginal treatment in early lactating (CC) and non-lactating (SC) llamas).

\begin{tabular}{lcc}
\hline Fase folicular & CC $(n=24)$ & SC $(n=23)$ \\
\hline Crecimiento $(n=16)$ & $8,1 \pm 0,4$ & $8,4 \pm 0,4$ \\
Estática $(n=16)$ & $7,0 \pm 0,4^{\mathrm{a}}$ & $11,2 \pm 0,5^{\mathrm{b}}$ \\
Regresión $(\mathrm{n}=15)$ & $6,0 \pm 0,5^{\mathrm{a}}$ & $9,4 \pm 0,4^{\mathrm{b}}$ \\
\hline
\end{tabular}

${ }^{\mathrm{ab}}$ valores con letra diferente en la misma fila difieren significativamente $(p<0,05)$. 
Tabla II. Duración (días; media $\pm E$ EM) de la regresión del folículo dominante en fases de crecimiento y estática folicular en llamas con cría (CC) y sin cría (SC) tratadas con AMP intravaginal. (Regression period (days; mean \pm SEM) of the dominant follicle in growing and static phases in early lactating (CC) and non-lactating (SC) llamas treated with intravaginal MPA).

\begin{tabular}{lcccc}
\hline $\begin{array}{l}\text { Estado lactacional } \\
\text { Fase folicular }\end{array}$ & \multicolumn{2}{c}{$\mathrm{CC}(\mathrm{n}=15)$} & \multicolumn{2}{c}{$\mathrm{SC}(\mathrm{n}=12)$} \\
Crecimiento $(\mathrm{n}=7)$ & Estática $(\mathrm{n}=7)$ & Crecimiento $(\mathrm{n}=5)$ & Estática $(\mathrm{n}=7)$ \\
\hline Regresión (días) & $4,1 \pm 0,5^{\mathrm{b}}$ & $3,5 \pm 0,5^{\mathrm{b}}$ & $3,5 \pm 0,9^{\mathrm{b}}$ & $6,0 \pm 0,5^{\mathrm{a}}$ \\
\hline
\end{tabular}

${ }^{a b}$ Valores con letra diferente en la misma fila difieren significativamente $(p<0,05)$.

tró que el día de inicio de la regresión del FD1 no fue afectado significativamente $(\mathrm{p}>0,05)$ por el uso de BE, la FFI, ni por la interacción entre factores. Sin embargo, fue observado un efecto $(\mathrm{p}<0,05)$ del EL (con cría $=2,0 \pm 04, \mathrm{n}=15$ y sin cría $1,1 \pm 0,3, \mathrm{n}=12$; media \pm EEM).

Se observó interacción $(\mathrm{p}=0,015)$ entre el EL y la FFI sobre la duración de la regresión (tabla II), debido a una mayor duración en hembras sin cría en FFI estática. Sin embargo, la tasa de regresión no fue $(\mathrm{p}>0,05)$ afectada por los factores principales ni por sus interacciones, por lo tanto, la tasa de

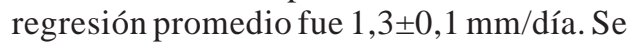
observó una relación positiva entre el diámetro del FD1 al día 0 y la duración de la regresión ( $\mathrm{p}<0,001)$ (figura 1).

Parámetros del FD2. En ninguno de los parámetros analizados del FD2 se observó efecto de la FFI, por lo tanto, los resultados fueron agrupados según el EL y el uso de $\mathrm{BE}$. En consecuencia, el número de unidades experimentales fue mayor en cada factor principal analizado.

El día de emergencia del FD2 no fue afectado $(p>0,05)$ por el EL ni la interacción entre factores principales, pero fue significativamente $(\mathrm{p}<0,05)$ diferente entre las llamas tratadas o no con BE (tabla III). El diámetro del FD2 al día 12 de comenzado el experimento fue afectado $(\mathrm{p}<0,05)$ por el uso de BE y el EL, siendo menor en las llamas tratadas con BE y en las llamas con cría al pie (tabla III). No se observó efecto significativo $(p>0,05)$ de los factores principales ni interacción sobre la tasa de crecimiento del FD2, por lo tanto, la tasa de crecimiento promedio ( \pm EEM) fue $0,7 \pm 0,1 \mathrm{~mm} /$ día.

Respuesta al tratamiento con AMP para sincronización e inducción de la ovulación. El porcentaje de hembras que presentó un FD2 $\geq 6 \mathrm{~mm}$ al día 12 no fue afectado por el EL, la FFI ni las interacciones entre factores principales. Sin embargo, se observó un efecto significativo $(\mathrm{p}<0,05)$ del uso de BE (tablaIV).

La tasa ovulatoria del FD2 ( $\mathrm{n}^{\circ}$ de hembras que ovularon/no. de hembras con FD2) no fue afectada $(\mathrm{p}>0,05)$ por el uso de BE ni por el EL. En cambio, la tasa ovulatoria total ( $\mathrm{n}^{\circ}$ de hembras que ovularon $/ \mathrm{n}^{\circ}$ de hembras con FD2 más $n^{\circ}$ de hembras con folículo persistente) fue afectada $(\mathrm{p}<0,05)$ por el uso de BE (tabla IV).

Concentración de hormonas plasmáticas. La interacción día por tratamiento fue significativa $(\mathrm{p}<0,05)$ para la concentración media de $\mathrm{E}_{2}$. La concentración plasmática de $E_{2}$ fue mayor $(p<0,05)$ entre los días 1 y 5 en hembras tratadas con $\mathrm{BE}$ con respecto a las hembras no tratadas, pero esta diferencia no fue observada desde el día 6 en adelante (figura 2).

No se observó efecto $(p>0,05)$ de los factores principales ni interacción sobre la concentración plasmática de progesterona determinada ocho días después de la ovula- 
Tabla III. Emergencia (día; media \pm EEM) y diámetro ( $m$ m; media $\pm E E M)$ del FD2 al momento de la inducción de la ovulación (día 12) en llamas con cría (CC) y sin cría (SC) tratadas con AMP intravaginal. (DF2 emergence (day; mean \pm SEM) and diameter ( $\mathrm{mm}$; mean \pm SEM) at the time of ovulation induction (day 12) in early lactating (CC) and non-lactating (SC) llamas treated with intravaginal MPA).

\begin{tabular}{lcccccc}
\hline & \multicolumn{3}{c}{ Día de emergencia } & \multicolumn{3}{c}{ Diámetro del FD2 (día 12) } \\
& CC $(n=16)$ & SC $(n=17)$ & Total $(n=33)$ & CC $(n=13)$ & SC $(n=15)$ & Total $(n=28)$ \\
\hline Con BE $(n=15)$ & $7,1 \pm 0,8$ & $6,3 \pm 0,8$ & $6,5 \pm 0,6^{\mathrm{a}}$ & $5,7 \pm 0,5$ & $7,3 \pm 0,4$ & $6,5 \pm 0,3^{\mathrm{a}}$ \\
Sin BE $(\mathrm{n}=18)$ & $4,9 \pm 0,7$ & $4,1 \pm 0,7$ & $4,2 \pm 0,5^{\mathrm{b}}$ & $7,5 \pm 0,4$ & $8,6 \pm 0,4$ & $8,0 \pm 0,3^{\mathrm{b}}$ \\
Total $(\mathrm{n}=33)$ & $5,9 \pm 0,6$ & $4,9 \pm 0,5$ & - & $6,7 \pm 0,3^{\mathrm{c}}$ & $7,9 \pm 0,3^{\mathrm{d}}$ & - \\
\hline
\end{tabular}

${ }^{a b}$ Valores con letra diferente en la misma columna difieren significativamente $(p<0,05)$.

${ }^{c d} V$ alores con letra diferente en la misma fila difieren significativamente $(p<0,05)$.

ción inducida, en consecuencia, la concentración plasmática promedio $( \pm \mathrm{EEM})$ fue $4,3 \pm 0,2 \mathrm{ng} / \mathrm{ml}$ (rango= 1,8 a $6,3 \mathrm{ng} / \mathrm{ml})$.

\section{DISCUSIÓN}

Los camélidos sudamericanos son especies cuya ovulación es inducida por la cópula, y el desarrollo de un cuerpo lúteo (fase luteal) después de una cópula infértil o durante la gestación deprime la dinámica folicular ovárica pero no la inhibe. En cambio, el uso de $\mathrm{P}_{4}$ inyectable (Alberio y Aller, 1996) o intravaginal (Chaves et al., 2002) logró inducir la atresia de los folículos presentes en diferentes fases, observados por medio de ultrasonografía en llamas no lac-

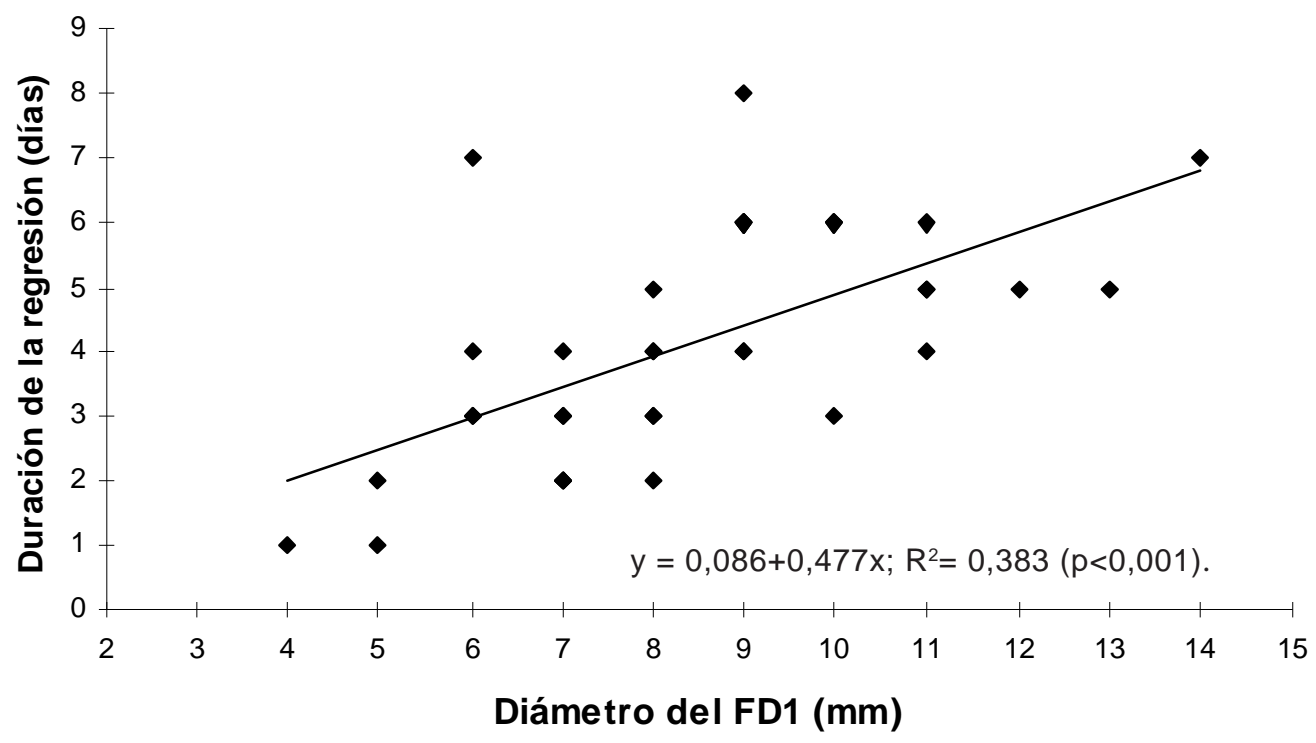

Figura 1. Relación lineal entre el diámetro del folículo dominante al comienzo del tratamiento intravaginal con AMP y la duración de la fase de regresión. (Lineal relation between dominant follicle diameter at the start of the MPA intravaginal treatment and the regression period).

Archivos de zootecnia vol. 60, núm. 232, p. 1014. 
Tabla IV. Efecto del estado lactacional y el tratamiento con BE sobre la dinámica folicular y ovulación en llamas con cría (CC) y sin cría (SC) tratadas con AMP intravaginal. (Effect of lactational status and EB treatment on the follicular dynamics and ovulation in early lactating (CC) and non-lactating (SC) llamas treated with intravaginal MPA).

\begin{tabular}{|c|c|c|c|c|}
\hline & \multicolumn{2}{|c|}{$C C(n=24)$} & \multicolumn{2}{|c|}{$S C(n=23)$} \\
\hline & $\begin{array}{l}\text { con BE } \\
(n=12)\end{array}$ & $\begin{array}{l}\sin B E \\
(n=12)\end{array}$ & $\begin{array}{l}\text { con BE } \\
(n=12)\end{array}$ & $\begin{array}{l}\sin B E \\
(n=11)\end{array}$ \\
\hline № hembras con FD1 en regresión (\%) & $12(100)$ & $11(91,7)$ & $10(83,3)$ & $9(81,9)$ \\
\hline $\mathrm{N}^{\circ}$ hembras con emergencia del FD2 (\%) & $7(58,3)$ & $9(75,0)$ & $8(66,7)$ & $9(81,8)$ \\
\hline $\mathrm{N}^{0}$ hembras con FD2 $\geq 6$ mm (día 12) (\%) & $4(57,1)^{a}$ & $9(100)^{b}$ & $6(75,0)^{a}$ & $9(100)^{b}$ \\
\hline No hembras con ovulación del FD2 & 3 & 8 & 6 & 9 \\
\hline Tasa ovulatoria del FD2 (\%) & 75,0 & 88,9 & 100,0 & 100,0 \\
\hline № hembras con folículo persistente (\%) & $0(0)$ & $1(8,3)$ & $2(16,7)$ & $2(18,2)$ \\
\hline No hembras con ovulación del FP & 0 & 0 & 2 & 2 \\
\hline Tasa ovulatoria del folículo persistente (\%) & 0 & 0 & 100,0 & 100,0 \\
\hline $\mathrm{N}^{0}$ total de hembras que ovularon & 3 & 8 & 8 & 11 \\
\hline Tasa ovulatoria total (\%) & $25,0^{\mathrm{b}}$ & $66,7^{\mathrm{a}}$ & $66,7^{\mathrm{b}}$ & $100,0^{\mathrm{a}}$ \\
\hline
\end{tabular}

${ }^{a b} V$ alores con letra diferente en la misma fila dentro de cada estado lactacional difieren significativamente $(p<0,05)$.

tando. Sin embargo, no hay información sobre el uso de estos tratamientos en hembras en lactancia temprana.

En bovinos, está demostrado que el tratamiento con $\mathrm{P}_{4}$ combinado con $\mathrm{E}_{2}$ induce la regresión del $\mathrm{FD}$ y la emergencia de una nueva onda folicular en forma sincrónica (Bó et al., 1993; 1995). En base a estas observaciones, en el presente trabajo se realizó un tratamiento con BE inyectable al inicio de un tratamiento con progestágenos (AMP intravaginal e inyectable) para estudiar su efecto sobre la dinámica folicular en llamas en post-parto temprano en diferentes fases foliculares al momento de comenzar el tratamiento.

Dinámica folicular previa al tratamiento. La dinámica del FD observada por medio de ecografía los tres días previos al comienzo del tratamiento fue similar al informado por otros autores (Adams et al., 1990; Bravo et al., 1990), el cual permitió determinar tres subgrupos de acuerdo a la fase de desarrollo (crecimiento, estática o regresión). Por otro lado, en el presente estudio, los diáme- tros foliculares en llamas en lactancia estuvieron de acuerdo con lo informado previamente (Adams et al., 1990, Ratto et al., 2003). Ambos grupos de investigación, observaron que la lactancia estuvo asociada a un menor diámetro máximo del FD en comparación con las hembras sin cría. Esto coincide con lo observado en vacas con cría, donde el diámetro máximo del FD es menor, debido a una menor concentración de $\mathrm{LH}$ causada por una menor frecuencia en su secreción pulsátil (Short et al., 1990).

Dinámica del FD1 durante el tratamiento. En llamas sin cría, se observó una mayor duración de la regresión del FD1 en fase estática, probablemente porque éstas presentan folículos de mayor diámetro y ondas más largas que las hembras en lactancia (Adams et al., 1990). En el presente estudio, el FD1 en fase estática alcanzó su mayor diámetro (11,2 mm) al comienzo del tratamiento con AMP y requirió mayor tiempo para llegar nuevamente a $3 \mathrm{~mm}$. Estos resultados concuerdan con lo observado en alpacas por Vaughan et al. (2004), quie- 
nes demostraron que la duración del intervalo entre ondas tiene una relación positiva con el diámetro del FD, es decir, cuanto mayor es el diámetro del FD más tiempo demoraría en regresar y por consiguiente retrasaría la emergencia de la nueva onda folicular.

El tratamiento hormonal progestativo (AMP intravaginal e inyectable) utilizado en este ensayo para el control de la dinámica folicular, indujo la regresión del FD1 presente al inicio del tratamiento en la mayoría de las hembras tratadas y la tasa de regresión promedio determinada fue menor a la observada en ausencia de un tratamiento progestativo, la cual fue de 1,6 mm/día (Aller y Alberio, 1996).

En los animales que se observó un FP, el tratamiento con AMP no indujo la regresión del FD1, el cual continuó su desarrollo hasta el momento de la inducción de la ovulación (día 12) y ejerció su dominancia inhibiendo la emergencia de una nueva onda folicular, probablemente, porque estos folículos secretan gran cantidad de $\mathrm{E}_{2}$ que mantienen baja la concentración de FSH (Viñoles et al., 1999). En exámenes realizados por medio de laparoscopía (Bravo y Sumar, 1989), el folí-

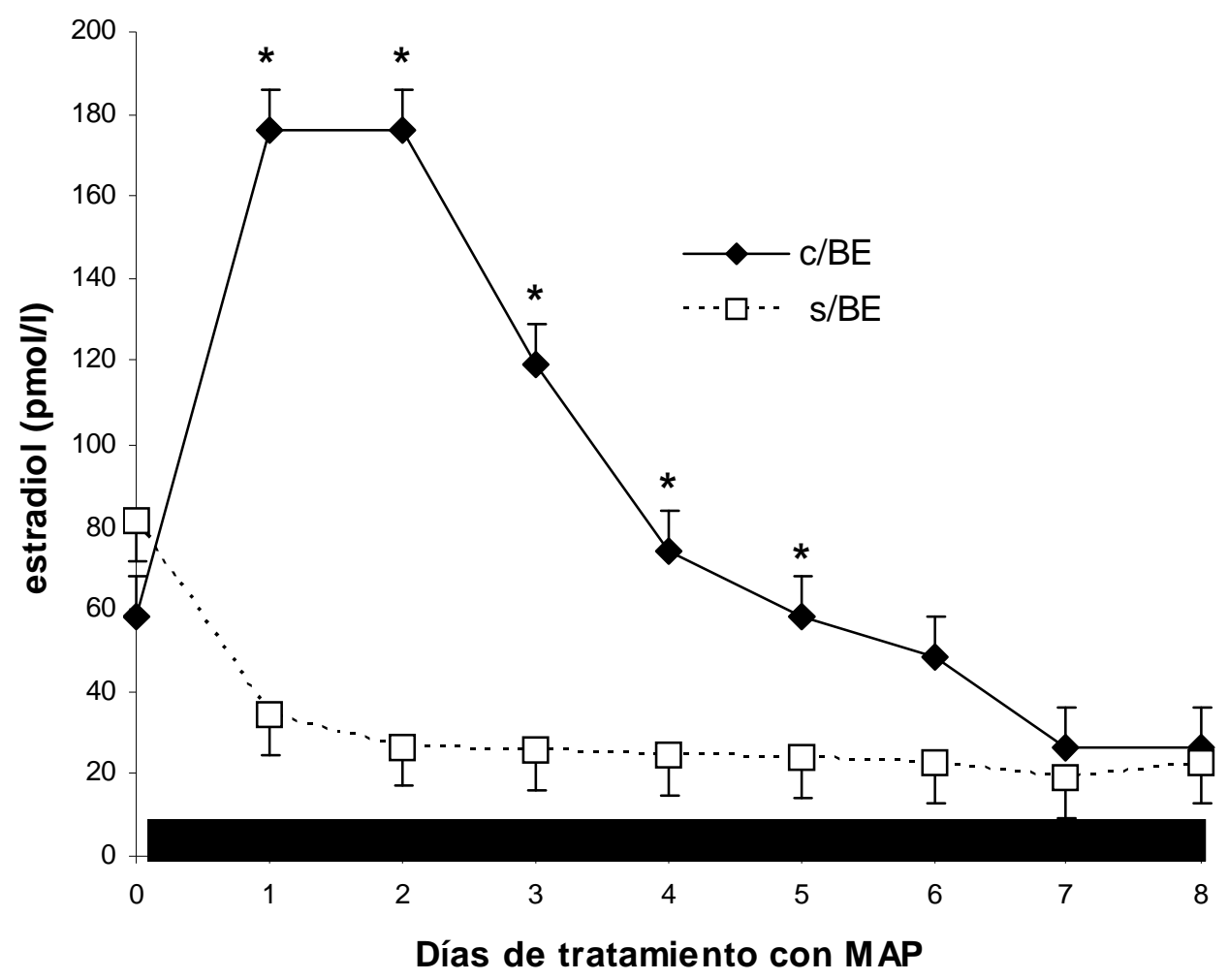

Figura 2. Concentración (media $\pm E E M$ ) plasmática de $17 \beta$-estradiol durante un tratamiento de 8 dias con esponja intravaginal impregnada con $150 \mathrm{mg}$ de MAP combinada o no con BE $\left({ }^{*} p<0,05\right)$. Barra sólida indica la duración del tratamiento intravaginal. (Plasma estradiol$17 \beta$ concentrations (mean \pm SEM) during an intravaginal MPA treatment for 8 days combined with (c/BE) or without $(\mathrm{s} / \mathrm{BE})$ estradiol benzoate $\left({ }^{*} \mathrm{p}<0,05\right)$. The solid bar indicates when intravaginal sponge was in situ). 
culo que presentó un diámetro >12 mm fue considerado quístico. Sin embargo, en este estudio, los folículos persistentes no superaron los $12 \mathrm{~mm}$ y tuvieron una respuesta ovulatoria de $80 \%(4 / 5)$, con la posterior formación de un CL funcional. En el bovino, el FD ovulatorio que persiste más de 10 días (FD persistente) no parece tener afectada su habilidad ovulatoria ni la subsiguiente formación luteal, pero el ovocito está envejecido y su fertilidad reducida (Mihm et al., 1994).

Dinámica folicular del FD2 y estradiol plasmático. La tasa promedio de crecimiento del FD2 no fue afectada por ninguno de los efectos principales y es coincidente con las observaciones de Adams et al. (1990), pero menor a la observada por Aller y Alberio (1996) en condiciones fisiológicas (1,1 mm/ día). Este resultado indicaría que el tratamiento con AMP utilizado en este estudio no afectó la emergencia ni el desarrollo de la nueva onda folicular, en coincidencia con lo observado en llamas con cría o sin cría tratadas con distintos métodos de sincronización folicular (Ratto et al., 2003).

La determinación de la concentración de $\mathrm{E}_{2}$ en hembras tratadas con AMP en combinación o no con $\mathrm{BE}$, fue empleada en este ensayo para observar la relación entre la concentración plasmática y los cambios foliculares. En llamas, está demostrado que la progesterona, tanto del cuerpo lúteo como exógena (Adams et al., 1990), y los progestágenos (Aba et al., 1999; Chaves et al., 2002) ejercen un efecto depresor sobre la actividad folicular y la producción de $\mathrm{E}_{2}$. En este ensayo, en las hembras sin $\mathrm{BE}$, se observó una rápida disminución en la concentración de $\mathrm{E}_{2}$ a las 24 horas, manteniéndose en niveles bajos durante el tratamiento con AMP. Esta disminución en la producción de $\mathrm{E}_{2}$ estaría de acuerdo con lo informado por Aba et al. (1999), quienes demostraron el efecto depresor del progestágeno (esponjas intravaginales impregnadas con $120 \mathrm{mg}$ de AMP durante 9 días) sobre la producción de $\mathrm{E}_{2}$ hasta el $5^{\circ}$ día desde su inserción. Similar efecto se observó hasta el $7^{\circ}$ día en llamas tratadas con progesterona (CIDR: 0,33 g) durante 16 días (Chaves et al., 2002), seguido de un incremento lento, a pesar del tratamiento. Por otro lado, Aba et al. (2005) trabajando con vicuñas tratadas con CIDR (0,33 g) durante 5 días, observaron una concentración mínima de $\mathrm{E}_{2}$ recién en el día 4 del tratamiento.

Trabajos previos demostraron que esponjas intravaginales impregnadas con progestágenos a distintas dosis (AMP= 120,240 y $480 \mathrm{mg}$ ) no inhibieron ni sincronizaron la actividad folicular (Ferrer et al., 1999), ya que el diámetro máximo del FD se mantuvo por encima de $7 \mathrm{~mm}$ durante todo el tratamiento (13 días). Sin embargo, en el presente experimento, el tratamiento con esponja conteniendo $150 \mathrm{mg}$ de AMP y la inyección de $5 \mathrm{mg}$ de AMP, indujo la regresión del FD1, probablemente debido al efecto del progestágeno que disminuye la frecuencia pulsátil de LH, puesto que la LH estimula el crecimiento del FD y promueve la aromatización de los andrógenos a $E_{2}$ en las células de la granulosa (Adams et al., 1992). En cambio, en las llamas tratadas con BE, la regresión y la atresia del FD1 se debió al feed-back negativo del estradiol combinado con el progestágeno sobre la secreción de LH, tal como se informara en vacas (Burke et al., 1998), demostrando que el estradiol potencia la habilidad de la progesterona para suprimir la LH incrementando la sensibilidad del hipotálamo o hipófisis al efecto inhibitorio de la progesterona (Burke et al., 1996).

En un estudio en alpacas, la administración de una sola inyección de estradiol (0,5 ó $2 \mathrm{mg}$ ) indujo la regresión de los folículos ováricos al $4^{\circ}$ día del tratamiento, independientemente de la fase folicular presente al momento del mismo y con la posterior emergencia de una nueva onda folicular (día 6) y subsiguiente desarrollo de un nuevo folículo dominante preovulatorio (día 10-11) (D'Occhio et al., 1998). En cambio, Ratto et al. (2003) empleando progesterona-estra- 
diol inyectables no concluyeron claramente acerca del efecto de estos esteroides sobre la emergencia sincrónica de la onda folicular, pero sugirieron que la sincronización folicular no debe ser evaluada por la respuesta ovulatoria per se, tal como la consideran algunos autores, ya que la tasa ovulatoria no difiere entre hembras sincronizadas y no sincronizadas. De manera que, existe información inconsistente acerca del efecto de los distintos tratamientos sobre la dinámica folicular en los camélidos.

En el presente estudio, las hembras que recibieron $\mathrm{BE}$ presentaron un rápido incremento y posterior disminución en la concentración plasmática de estradiol, acompañada por la atresia del FD1 y por la emergencia del FD2 (día=6,5), independientemente de la fase folicular inicial. En bovinos, se demostró que el intervalo desde el momento de la inyección de BE hasta la emergencia del FD2 es dependiente de la dosis de BE (Lane et al., 2001) y la duración de la supresión de FSH es transitoria y depende del pico de concentración de $\mathrm{E}_{2} \mathrm{y}$ del tiempo que tarda en llegar a niveles basales. De acuerdo a estas consideraciones, puede inferirse que la administración de 2 mg de BE en llamas de $108 \mathrm{~kg}$ de peso vivo promedio (1 mg/54 kg) utilizadas en este estudio, pudo haber sido una dosis demasiado alta para la especie, tal que, dicha dosis llevó a niveles bajos de $E_{2}$ recién al día 6 , causando una mayor duración en la supresión de FSH y por lo tanto una demora en la emergencia de la nueva onda folicular. En las llamas no tratadas con BE, la emergencia del FD2 ocurrió dos días antes que en las llamas tratadas. Posiblemente, una razón fisiológica del por qué, en ambos tratamientos (con y sin $\mathrm{BE}$ ), el incremento de $\mathrm{E}_{2}$ correspondiente a la emergencia y desarrollo del FD2 no fue detectada, podría deberse a que el folículo en crecimiento estaría bajo el efecto del AMP liberado por la esponja que simuló una fase luteal, deprimiendo su crecimiento y la producción de estrógenos hasta el retiro de la misma.
Ovulación y formación del CL. La ovulación y el desarrollo del CL ocurre solamente como respuesta a la cópula o estímulo hormonal sobre folículos maduros. En general, los estímulos hormonales presentan una eficiencia entre $80-100 \%$ en la tasa de ovulación, siempre y cuando el folículo preovulatorio se encuentre en fase de crecimiento o maduración con un diámetro $\geq 6 \mathrm{~mm}$ (Bravo et al., 1991). En este trabajo se estableció previamente que las hembras que presentaran un folículo $\geq 6 \mathrm{~mm}$ el día 12 serían sometidas al estímulo de la GnRH.

Del total de hembras con FD2 ovulatorio ( $\geq 6 \mathrm{~mm}$ ), el $93 \%$ ovuló (hasta las 48 horas post-GnRH). Este resultado fue mayor a los obtenidos por Aller et al. (1999) (70,5\%) y Ferrer et al. (2002) (62,5\%) en llamas. La diferencia observada en la tasa ovulatoria del FD2, entre las llamas tratadas con BE (37,5\%) y las no tratadas (73,9\%), considerando el total de llamas de cada grupo, sería consecuencia del retraso de dos días en el día promedio de emergencia en las llamas tratadas con BE. En consecuencia, para mejorar la tasa ovulatoria cuando se administra BE, la ovulación debiera ser inducida al menos dos días más tarde para obtener un mayor diámetro del FD2 en la mayoría de las hembras. Sin embargo, si se observa la tasa ovulatoria per se, es decir, la tasa ovulatoria delFD2 $\geq 6 \mathrm{~mm}$, el tratamiento con BE $(90,0 \%)$ fue similar a sin BE $(94,4 \%)$, por lo cual, la administración de BE en este ensayo no afectó la tasa ovulatoria del FD2. La tasa ovulatoria promedio, coincide con lo informado por Ratto et al. (2003), quienes concluyeron que los tratamientos de sincronización no afectan la tasa de ovulación luego de un estímulo por monta natural a tiempo fijo.

La posterior formación de un CL, fue confirmada mediante el nivel plasmático de $\mathrm{P}_{4}$ obtenido a los 8 días post-GnRH. Valores superiores a $0,4 \mathrm{ng} / \mathrm{ml}$ reflejan actividad luteal (Bravo et al., 1991) y los valores máximos se encuentran al día 8 post-estímu- 
lo (Sumar et al., 1988) ó 6 días post-ovulación (Adams et al., 1991). Por lo tanto, el FD2 con un diámetro $\geq 6 \mathrm{~mm}$, tendría la capacidad de ovular y desarrollar un CL normal, pero tal vez sólo cuando se encuentre en crecimiento. En cambio, la inducción de folículos en regresión induce la formación de un CL de menor duración o estructuras luteinizadas (Bravo et al., 1991).

En conclusión, la FFI afectó la dinámica de la regresión del FD1 pero no tuvo influencia sobre la emergencia y el desarrollo de la nueva onda folicular. Sin embargo, el BE utilizado al comienzo de un tratamiento con AMP (intravaginal e inyectable) para el control de la dinámica folicular, demoró la emergencia de la nueva onda folicular aproximadamente en dos días, comparado con el tratamiento sin BE. Como consecuencia de

\section{BIBLIOGRAFÍA}

Aba, M.A. 1995. Studies on the reproductive endocrinology of llamas and alpacas, from mating troughout early pregnancy. Tesis Master of Science. SLU. Faculty of Veterinary Medicine. Uppsala. Sweden. 78 pp.

Aba, M.A., Quiroga, M.A., Auza, N., Forsberg, M. and Kindahl, H. 1999. Control of ovarian activity in Ilamas (Lama glama) with medroxiprogesterone acetate. Reprod. Dom. Anim., 34: 471-476.

Aba, M.A., Miragaya, M.H., Chaves, M.H., Capdevielle, E.F., Rutter, B. and Agüero, A. 2005. Effect of exogenous progesterone and eCG treatment on ovarian follicular dynamics in vicunas (Vicugna vicugna). Anim. Reprod. Sci., 86: 153-161.

Adams, G., Sumar, J. and Ginther, O. 1990. Effects of lactational and reproductive status on ovarian follicular waves in llamas. J. Reprod. Fertil., 90: 535-545.

Adams, G., Sumar, J. and Ginther, O. 1991. Form and function of the corpus luteum in llamas. Anim. Reprod. Sci., 24: 127-138.

Adams, G., Matteri, R.L. and Ginther, O. 1992. Effect of progesterone on ovarian follicles, emergence of follicular waves and circulation lo anterior, el diámetro del FD2 al momento de la inducción de la ovulación fue menor que el observado en las llamas que no recibieron BE. Por otro lado, la lactancia no afectó al momento de emergencia de la nueva onda folicular, pero en este estado reproductivo las hembras presentaron un menor diámetro del FD2 al momento de la inducción de la ovulación. Sin embargo, la tasa ovulatoria del FD2 fue similar en ambos estados reproductivos.

\section{AGRADECIMIENTOS}

Al Técnico Agr. René H. Cabrera (EEA INTA Abra Pampa) por su asistencia en los trabajos de campo. Este trabajo se realizó gracias al aporte económico otorgado por el Instituto Nacional de Tecnología Agropecuaria.

follicle-stimulating hormone in heifers. J. Reprod. Fertil., 95: 627-640.

Alberio, R. y Aller, J. 1996. Control y sincronización de la onda folicular mediante aplicación de progesterona exógena en llamas. Rev. Arg. Prod. Anim., 16: 325-329.

Aller, J. y Alberio, R. 1996. Dinámica folicular en llamas en la época otoño-invernal. Rev. Arg. Prod. Anim., 16: 319-323.

Aller, J., Cancino, A., Rebuffi, G. y Alberio, R. 1999. Inducción de la ovulación en llamas. II Congreso Mundial sobre Camélidos. Cusco. Perú. 4-7 Nov. p. 91.

Bó, G.A., Adams, G.P., Nasser, L.F., Pierson, R.A. and Mapletoft, R.J. 1993. Effect of estradiol valerate on ovarian follicles, emergence of follicular waves and circulating gonadotropins in heifers. Theriogenology, 40: 225-239.

Bó, G.A., Adams, G.P., Caccia, M., Martinez, M., Pierson, R.A. and Mapletoft, R.J. 1995. Ovarian follicular wave emergence after treatment with progestogen and estradiol in cattle. Anim. Reprod. Sci., 39: 193-204.

Bravo, P. W. and Sumar J. 1989. Laparoscopic examination of the ovarian activity in alpacas. Anim. Reprod. Sci., 21: 271-281. 
Bravo, P. W., Fowler M.E., Stabenfeldt, G.H. and Lasley B.L. 1990. Ovarian follicular dynamics in the Llama. Biol. Reprod., 43: 579-585.

Bravo, P., Stabendfeldt, G., Lasley, B. and Fowler, M. 1991. The effect of ovarian follicle size on pituitary and ovarian responses to copulation in domesticated South American camelids. Biol. Reprod., 45: 553-559.

Burke, C. R., Macmillan, K. L. and Boland, M. P. 1996. Oestradiol potentiates a prolonged progesterone-induced suppression of $\mathrm{LH}$ release in ovariectomised cows. Anim. Reprod. Sci., 45: 13-28.

Burke, C. R., Morgan, S., Clark, B.A. and Rhodes, F.M. 1998. Effect of luteolysis on control of ovarian follicles using oestradiol benzoate and progesterone in cattle. Proc. N.Z. Soc. Anim. Prod., 58: 89-91.

Carretero, M.I., Chaves, M.G., Agüero, A. y Miragaya, M. 2006. Sincronización de la onda folicular mediante la administración de progesterona y benzoato de estradiol inyectables en la especie Lama glama. IV Congreso Mundial sobre Camélidos. Santa María. Catamarca. Argentina. 11-15 Oct. pp. 38-42.

Cavilla., M.V., Bianchi, C.P., Waimann, E., Aguilera, M.F. y Aba, M. 2006. Perfil de progesterona plasmática y actividad folicular en llamas tratadas con un dispositivo intravaginal comercial conteniendo 0,78 gramos de progesterona. IV Congreso Mundial sobre Camélidos. Santa María. Catamarca. Argentina. 11-15 Oct. pp. 43-48.

Chaves, M.G., Aba, M., Agüero, A., Egey, J., Berestin, V. and Rutter, B. 2002. Ovarian follicular wave and the effect of exogenous progesterone on follicular activity in non-mated llamas. Anim. Reprod. Sci., 69: 37-46.

D'Occhio, M J. and Novoa, C. 1998. Current evaluation of new breeding concept could enhance the efficiency of mating and genetic improvement in alpaca. Town and Country Farmer, 35: 37.

Ferrer, M.S., Agüero, A., Flores, M. y Rutter, B. 1999. Control de la dinámica folicular en la llama (Lama glama) utilizando esponjas intravaginales con medroxiprogesterona. II Congreso Mundial sobre Camélidos. Cusco. Perú. 4-7 Nov. p. 173. Ferrer, M.S., Agüero, A., Chaves, M.G., Russo, A. F. y Rutter, B. 2002. Sincronización de la onda folicular mediante el uso de buserelina en la llama (Lama glama). InVet, 4: 7-11.

Lane, E.A., Austin, E.J., Roche, J.F. and Crowe, M.A. 2001. The effect of estradiol benzoate or a synthetic gonadotropin-releasing hormone used at the start of a progesterone treatment on estrous response in cattle. Theriogenology, 56: 79-90.

Mihm, M., Baguisi, A., Boland, M.P. and Roche, J.F. 1994. Association between the duration of dominance of the ovulatory follicle and pregnancy rate in beef heifers. J. Reprod. Fertil., 102: 123-130.

Miragaya, M.H., Aba, M.A., Capdevielle, E.F., Ferrer, M.S., Chaves, M.G., Rutter, B. and Agüero, A. 2004. Follicular activity and hormonal secretory profile in vicuna (Vicugna vicugna). Theriogenology, 61: 663-671.

Ratto, M.H., Singh, J., Huanca, W. and Adams, G.P. 2003. Ovarian follicular wave synchronization and pregnancy rate after fixed-time natural mating in llamas. Theriogenology, 60: 16451656.

SAS. 1989. SASISTAT® User's Guide. Version 6. $4^{\text {th }}$ ed., vol. 2. SAS Institute Inc. Cary, NC. 846 pp.

Short, R.E., Bellows, R.A., Staigmiller, R.B., Berardinelli, J.G. and Custer, E.E. 1990. Physiological mechanisms controlling anestrus and infertility in postpartum beef cattle. J. Anim. Sci., 68: 799-816.

Sumar, J., Fredrisksson, G., Alarcon V., Kindahl, H. and Edqvist, L., 1988. Levels of 15-keto-13, 14-dihydro-PFG ${ }_{2 \alpha}$, progesterone and oestradiol$17 \beta$ after induced ovulations in llamas and alpacas. Acta Vet. Scand., 29: 339-346.

Vaughan J.L., Macmillan K.L. and D'Occhio M.J., 2004. Ovarian follicular wave characteristics in alpacas. Anim. Reprod. Sci., 80: 353-361.

Viñoles, C., Meikle, A., Forsberg, M. and Rubianes, E. 1999. The effect of subluteal levels of exogenous progesterone on follicular dynamics and endocrine patterns during the early luteal phase of the ewe. Theriogenology, 51: 1351-1361.

Archivos de zootecnia vol. 60, núm. 232, p. 1020. 